\title{
Sensing-based Spectrum Awareness in Cognitive Radio: Challenges and Open Research Problems
}

\author{
Miguel López-Benítez \\ Dept. Electrical Engineering and Electronics \\ University of Liverpool, United Kingdom \\ Email: M.Lopez-Benitez@liverpool.ac.uk
}

\begin{abstract}
Owing to the opportunistic nature of its operation principle, the performance of Dynamic Spectrum Access/Cognitive Radio (DSA/CR) systems depends on the spectrum occupancy pattern of primary systems. DSA/CR systems can monitor periodically the occupancy state of licensed channels in order to gain statistical information on their occupancy patterns, and exploit this information in decision-making processes. This paper provides an overview of relevant research topics related with the problem of providing DSA/CR systems with the ability to gain accurate, reliable and exploitable information on realtime spectrum occupancy. In particular, this paper reviews the following problems: i) spectrum sensing algorithms for spectrum occupancy estimation; ii) estimation of spectrum occupancy statistics based on spectrum sensing; and iii) statistical models for spectrum occupancy. Existing solutions are reviewed and open research problems are discussed as well.
\end{abstract}

Keywords-Cognitive radio; dynamic spectrum access; spectrum awareness; spectrum sensing.

\section{INTRODUCTION}

The Dynamic Spectrum Access (DSA) principle, based on the Cognitive Radio (CR) paradigm [1-5], is aimed at increasing the efficiency of spectrum utilisation by allowing unlicensed users (also referred to as secondary users) to access opportunistically some licensed bands temporarily and/or spatially unused by the legitimate licensed users (also referred to as primary users). Owing to the opportunistic nature of this spectrum access paradigm, the behaviour and performance of DSA/CR systems depends on the spectrum occupancy pattern of primary users. DSA/CR systems can benefit from an accurate knowledge of spectrum occupancy and its statistical properties. This knowledge can be exploited in spectrum and radio resource decision-making processes, leading to an increased DSA/CR system performance, a better interference protection of primary systems and a more efficient use of spectral resources. Some examples include the prediction of future trends in the spectrum occupancy $[6,7]$, the selection of the most appropriate band/channel for DSA/CR operation [8$11]$, or the decision on future actions to optimise the DSA/CR performance and spectrum efficiency [12-16]. However, gaining accurate and reliable real-time information on spectrum occupancy is a challenging task for DSA/CR systems. To date, this important problem has not received the required attention and satisfactory solutions are still necessary. In this context, this paper provides an overview of relevant research topics related with the problem of providing DSA/CR systems with the ability to gain accurate, reliable and exploitable information on real-time spectrum occupancy. In particular, this paper reviews the problems of: i) spectrum sensing algorithms, methods and techniques in the context of spectrum occupancy estimation; ii) reliable and accurate estimation of spectrum occupancy statistics based on empirical observations (i.e., spectrum sensing); and iii) statistical models for characterising spectrum occupancy patterns. Existing solutions are reviewed and open research problems are discussed as well.

\section{SPECTRUM AWARENESS}

The spectrum awareness function of a DSA/CR system is in charge of obtaining relevant information and knowledge of the surrounding radio environment, including the identification of portions of the radio spectrum that are available for opportunistic use (spectrum holes or white spaces [17]) at any time [5]. A DSA/CR system can make use of various techniques to obtain spectrum awareness information [5]:

- Beacon signals: This method is based on the use of regional beacon signals that are broadcast in appropriate signalling channels in order provide real-time information on the primary system(s) present in a certain geographical area. According to this method, DSA/CR terminals detect the regional beacon signals to obtain information about the surrounding environment (e.g., primary systems available, spectrum occupancy information, etc.). This method assumes the existence of an agreement between the primary spectrum licensee and the secondary DSA/CR system, whereby the primary network shares real-time information with the secondary network regarding spectrum utilisation. This approach enables a perfect knowledge of spectrum usage in the DSA/CR system. However, it requires modifications to the existing primary network, which may not be possible for some legacy radio technologies or simply economically infeasible. Some examples of approaches based on the use of beacon signals can be found in [18-22].

- Geolocation databases: This approach is based on a centrally managed spectrum usage database that contains relevant information about the regional spectrum usage, including location of primary transmitters, coverage areas, frequencies of operation, transmission powers, radio technologies, operation requirements, etc. According to this method, the database provides relevant regional information to $\mathrm{DSA} / \mathrm{CR}$ terminals based on their current geographical location (hence the term geolocation database). DSA/CR terminals can obtain a wide range of accurate information from databases. However, this approach has some drawbacks: 1) the DSA/CR system needs to rely on an external system to obtain spectrum awareness information (this poses important challenges, not only from technical but also administrative, legal and regulatory points of view); 2) DSA/CR terminals need 
to implement geolocation methods such as GPS or triangulation (involving problems of location accuracy, indoor location, etc.); 3) the updating of the database information is not an instantaneous process, which makes databases an inadequate alternative for spectrum bands with dynamic occupancy patterns.

- Spectrum sensing: A simple method to identify spectrum opportunities is to scan a channel/band and determine, by means of appropriate signal processing methods, whether a primary signal is present [23]. The main advantage of spectrum sensing is that the DSA/CR system does not need to rely on any external system to obtain knowledge of the environment and is a well-suited alternative for spectrum bands with dynamic occupancy patterns. However, due to the unavoidable presence of noise and other degrading effects, spectrum sensing algorithms may fall into mistakes, meaning that spectrum sensing methods are not perfectly reliable.

Geolocation databases and beacon signals place on the primary system the responsibility of providing spectrum awareness to the secondary DSA/CR system. While spectrum occupancy information is reliable (it is provided by the primary system), these approaches need a communication means between primary and secondary systems, which may lead to problems of economical feasibility and compatibility issues with legacy systems. Geolocation databases and beacon signals require the deployment of external infrastructure and requires location information. On the other hand, spectrum sensing solely relies on local observations of the spectrum occupancy without the need of an external system providing spectrum awareness. While spectrum sensing leads to more complex secondary terminals, no modifications to the primary system are required (i.e., it is compatible with legacy systems) and infrastructure costs are notably lower. However, spectrum sensing is somewhat unreliable due to practical limitations.

This paper focuses on spectrum sensing based spectrum awareness methods and discusses several existing solutions as well as relevant open research problems.

\section{SPeCtrum SENSING Algorithms}

A significant number of signal detection methods, commonly referred to as spectrum sensing algorithms, have been developed in the context of DSA/CR to identify the presence of primary signals [24-28]. Existing solutions provide different trade-offs between the sensing time required for a certain detection performance, the algorithm complexity and computational cost as well as the signal detection capabilities. However, their practical applicability depends on the amount of primary signal information available to the signal detector.

Energy detection, also referred to as radiometric detection [29], declares a channel/band to be busy if the signal energy lies above a properly set decision threshold, or idle otherwise. Energy detection is simple and works irrespective of the signal to be detected, thus making it a good option when a DSA/CR user knows nothing about the primary signal or when complexity/computational cost is a major concern. Although a simple and widely applicable method, energy detection is highly susceptible to uncertainty in the noise power, and cannot distinguish a weak primary signal from noise or interference, which results in a rather poor detection performance.
More advanced detection methods that exploit primary signal characteristics (e.g., modulation, coding, pilot signals, preambles or synchronisation sequences) can be employed to attain a better detection performance. When the primary signal is known to the secondary user (including modulation type and order, pulse shape, packet format, etc.), the optimal detector in additive white Gaussian noise (AWGN) is the matched filter, which maximises the received Signal-to-Noise Ratio (SNR) [30, 31]. Matched filters require a detailed knowledge of the primary signal to be detected and perform poorly when this information is not accurate [32].

The cyclostationary feature detection method, which was originally proposed in $[33,34]$, exploits the cyclostationarity (i.e., built-in periodicity in the average and autocorrelation) of modulated signals that results from sine wave carriers, pulse trains, repeating spreading, hopping sequences or cyclic prefixes. By analysing the signal's cyclic autocorrelation function, the signal (which is cyclostationary with spectral correlation) can be distinguished from noise (which is wide-sense stationary with no correlation). Cyclostationary feature detection can discriminate a primary signal from interference or noise even under low SNR conditions, thus outperforming energy detection; however, it is more complex and requires the cyclic frequency of the signal's autocorrelation function to be known.

Covariance-based detection [35] does not require any prior information, but relies on the presumption that the primary signal received at the DSA/CR user is autocorrelated, which can be exploited to differentiate the primary signal from the (uncorrelated) noise. In the hypothetical worst case that the primary signal is uncorrelated (i.e., it appears as white noise), a covariance-based detection would fail. In practice, real signals are usually correlated due to the dispersive nature of real channels (moreover, correlation can be introduced by oversampling the received signal or using multiple receive antennas [35]). However, the performance of this method depends on the primary signal statistics. Moreover, covariancebased detection is a computationally complex method.

As it can be appreciated in the previous discussion, each spectrum sensing method requires a different amount of information on the primary signal. Matched filter detection is the optimum method when detailed information on the primary signal is available. Cyclostationary detection can be employed when the cyclic frequency of the primary signal is known. Covariance-based detection is in general a suitable method if the primary signal is known to be correlated, or if it is feasible to introduce correlation by means of oversampling or using multiple receive antennas. When nothing is known about the primary signal, energy detection can still be employed.

Despite its practical performance limitations, energy detection has gained popularity as a spectrum sensing technique for DSA/CR due to its simplicity and general applicability (no prior knowledge of the signal to be detected is required since it works irrespective of the signal format), as well as its low computational and implementation costs. The main drawback, however, is its well-known detection performance limitations. More advanced sensing methods (such as matchedfilter detection, feature detectors, covariance-based detectors, etc.) in general outperform the conventional energy detection scheme by exploiting specific signal properties, which not only assumes some prior knowledge of the primary signal, but also restricts the field of application to a specific signal format. The performance improvement is normally obtained at the expense of an increased algorithm complexity and computational cost. 
Recently, alternative methods based on variations of the energy detection scheme have been proposed, which provide a better detection performance while preserving a similar level of complexity, computational cost and field of application, therefore providing performance improvements at virtually no extra cost. An example of this type of solutions is presented in [36], where multiple spectrum sensing events are combined and processed smartly in order to provide a more accurate and reliable estimation on the channel occupancy state. Figure 1 compares the Receiver Operating Characteristic (ROC) curve for the Classical Energy Detection (CED) algorithm and the Improved Energy Detection (IED) algorithm proposed in [36], while Figure 2 compares their sample and computational complexities. As it can be observed from these results, the main interest of the IED scheme proposed in [36] relies on its ability to outperform the CED scheme (Figure 1) while preserving a similar level of complexity (Figure 2) as well as its general applicability regardless of the particular signal format or structure to be detected (IED is essentially based on energy detection). These results motivate the search of variations of the energy detection method able to improve the low detection performance of the CED scheme, while preserving a similar level of complexity and computational cost as well as its general field of applicability. Solutions of this type would improve the weak point of energy detection (i.e., low detection performance), without incurring in the costs and disadvantages of other more complex detection methods (i.e., high level of algorithm complexity and computational cost along with a limited field of applicability). The main idea of the IED scheme proposed in [36] is to combine several spectrum sensing events into a single sensing decision, but other ideas based on variations of the energy detection method can be explored as well, including for example dynamic detection thresholds, double/multiple detection thresholds, parallel application of multiple energy detectors with different configurations and combinations of their individual sensing decisions, etc. These and other variations of the energy detection method can be investigated as a part of future research work.

\section{Estimation OF SPECTRUM OCCUPANCY Statistics}

The main purpose of spectrum sensing is to determine whether a primary channel is idle/busy so that a DSA/CR system knows when the channel can be accessed opportunistically. However, the idle/busy information obtained from spectrum sensing can be further processed in order to obtain more elaborated information (the primary channel/spectrum activity statistics), which can be exploited in spectrum and radio resource decision-making processes of the DSA/CR system.

The activity statistics of a primary channel are initially unknown to the DSA/CR system but can be estimated based on spectrum sensing decisions. The estimation of the duration of the idle/busy periods of a channel based on spectrum sensing decisions is illustrated in Figure 3. The channel is sensed with a finite sensing period $T_{s}$. In every sensing event, the spectrum sensing algorithm makes a binary decision on the idle $\left(\mathcal{H}_{0}\right)$ or busy $\left(\mathcal{H}_{1}\right)$ state of the channel. The sequence of observed idle/busy states can be used to make an estimation of the durations of the idle/busy periods. In particular, when the observed channel state changes, the time interval elapsed since the last state change is computed as shown in Figure 3 (a) to make an estimation $\widehat{T}_{i}$ of the real period duration $T_{i}$ ( $i=0$ for idle periods, $i=1$ for busy periods). By means of an appropriate processing of the observed period
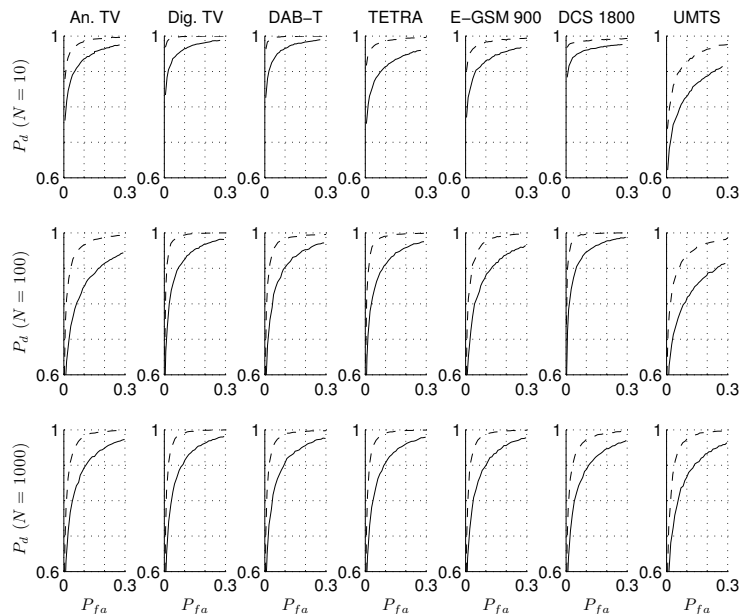

Fig. 1. Experimental ROC curve (i.e., detection probability $P_{d}$ versus false alarm probability $P_{f a}$ ) for the CED (solid line) and IED (dashed line) algorithms, for various radio access technologies and sensing periods $(N)$ [36].
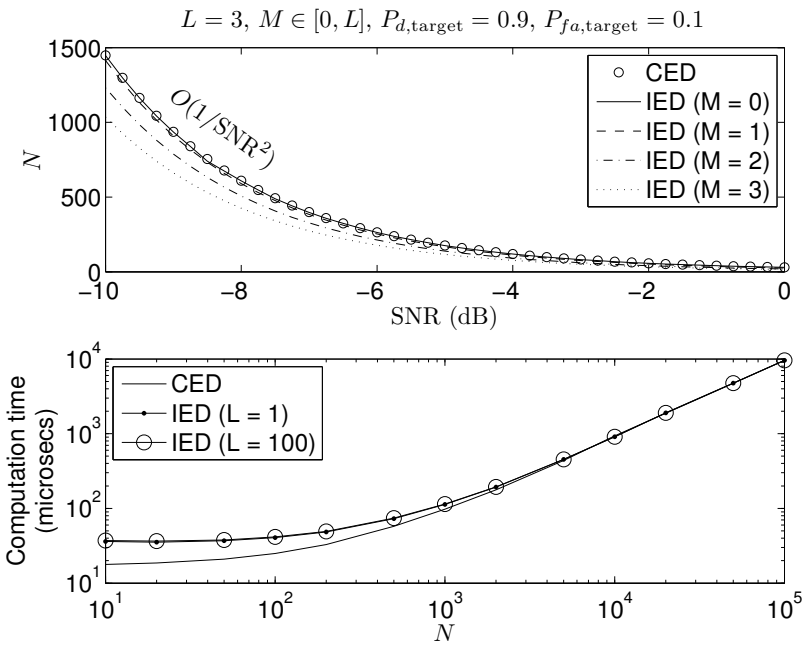

Fig. 2. Complexities of the CED and IED algorithms (upper graph: sample complexity; lower graph: computational complexity) [36].

durations, the DSA/CR system can make an estimation of the channel activity statistics such as the minimum period duration, the statistical moments (e.g., mean, variance, etc.) or the underlying distribution. However, the accuracy of the estimated statistics depends, among other factors, on the performance of the employed spectrum sensing method. When the primary Signal-to-Noise Ratio (SNR) at the DSA/CR receiver is sufficiently high, a Perfect Spectrum Sensing (PSS) scenario without sensing errors can be assumed. Unfortunately, spectrum sensing methods are imperfect in practice, meaning that some sensing errors may occur occasionally, thus leading to an Imperfect Spectrum Sensing (ISS) performance, especially under low SNR conditions. In particular, idle channels may sometimes be reported as busy (event referred to as false alarm), while busy channels may in some cases be reported as idle (missed detections). Sensing errors alter the sequence of idle/busy states observed in the channel and therefore affect the estimated period durations, which leads to incorrectly 


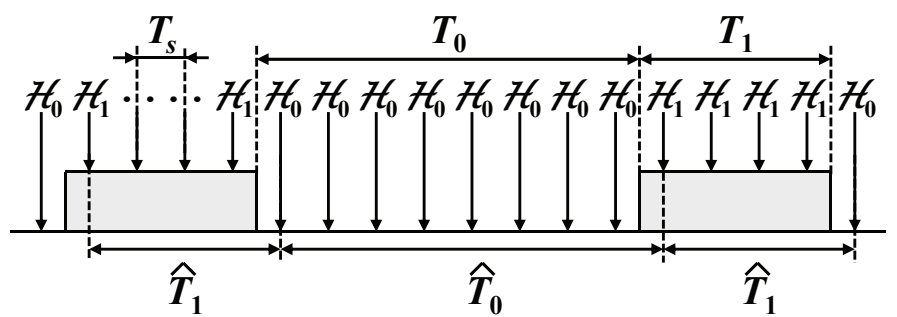

(a)

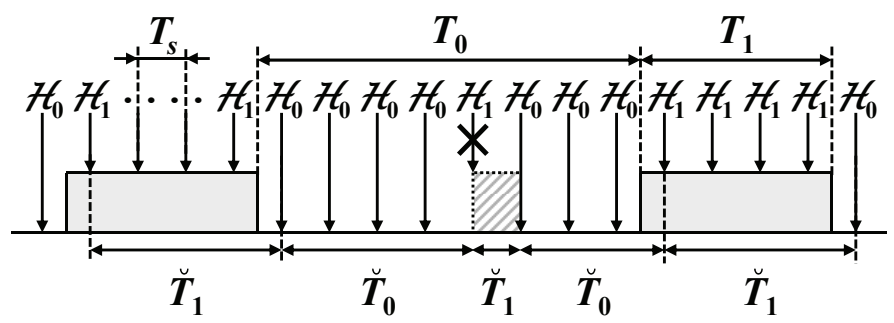

(b)

Fig. 3. Estimation of period durations from spectrum sensing decisions: (a) under perfect spectrum sensing, (b) under imperfect spectrum sensing [37].

estimated period durations $\breve{T}_{i}$. As shown in Figure 3(b), an idle period may be observed as a sequence of three periods (idle-busy-idle) as a result of a false alarm. Similarly, a missed detection may result in a busy period being reported as a sequence of busy-idle-busy periods. Incorrect period durations $\breve{T}_{i}$ may lead to inaccurate estimations of the channel activity statistics. As a result, sensing errors can severely affect the estimated channel activity statistics and their accuracy, thus limiting their practical utility.

In this context, an important research problem is the estimation of primary channel/spectrum activity statistics, based on empirical (spectrum sensing) observations, under the presence of practical limitations. Relevant aspects to be considered in this area include, but are not limited to:

- Relevant statistics: Some simple statistics about the primary channel/spectrum activity are the duration of the idle/busy periods and their minimum value, mean value, variance and underlying distribution. There are other more complex metrics that can be relevant (e.g., the channel duty cycle). However, such metrics can be obtained based on the aforementioned statistics [37].

- Practical limitations: Several important practical limitations that affect the estimation of channel/spectrum activity statistics based on sensing observations are:

- Imperfect sensing performance: The presence of missed detections and false alarms affect the estimated period durations as explained above.

- Finite sensing period: Spectrum sensing is performed with a certain periodicity $T_{s}$. The estimated period durations are integer multiples of $T_{s}$, which imposes a limit on the time accuracy to which period durations (and the subsequent statistics) can be estimated.

- Limited number of observations: The channel/spectrum activity statistics are computed based on a limited number of channel observations (sample size), which also imposes a limit on the accuracy of the estimated statistics.
- Aspects to be analysed: Some relevant questions to be answered in this area include, but are not limited to:

- What are the activity statistics actually estimated by a DSA/CR terminal?: Given the operation conditions (e.g., probability of missed detection/false alarm, sensing period, sample size, SNR, etc.), an important question is the analytical characterisation of the activity statistics that would observed by a DSA/CR terminal under such conditions.

- What is the difference between the estimated and the real channel activity statistics (i.e., the estimation error)?: Another important question is to what extent the activity statistics estimated by a DSA/CR terminal under certain operation conditions differ from the real statistics. The work reported in [37] provided some preliminary results/answers to this question based on a simulation-based approach.

- What can be done to minimise the estimation error?: Finally, an important question to address is how the estimation of channel/spectrum activity statistics can be made more accurate under the presence of degrading effects and practical limitations. The work reported in [37] proposed some simple methods to process the estimated period durations (based on the knowledge of the minimum period duration) in order to discard incorrectly estimated period durations and provide a more accurate estimation of the channel/spectrum activity statistics. However, this area still needs more sophisticated and effective solutions.

The work reported in [37] provided a first approach to above mentioned questions by performing a detailed simulation analysis on the impact of ISS performance on the estimation of primary activity statistics in DSA/CR systems. The relative importance of various parameters was analysed and quantified, and guidelines to properly configure the spectrum sensing function of DSA/CR systems were provided. Moreover, several methods to palliate the degrading effects of spectrum sensing errors on the estimated statistics were proposed and assessed as well. However, this area still requires a more rigorous and detailed study, including analytical solutions and experimental validations, thus leaving room for future research work.

\section{Modelling of Spectrum Occupancy Patterns}

The knowledge of the primary channel/spectrum activity statistics can be exploited in spectrum and radio resource decision-making processes of the DSA/CR system. Moreover, these statistics can also be incorporated into more comprehensive models that characterise the spectrum occupancy pattern of a spectrum band. Spectrum occupancy models can find several applications, ranging from analytical studies to the design and dimensioning of DSA/CR networks as well as the development of innovative simulation tools and more efficient DSA/CR techniques. In order to guarantee the realism and accuracy of such models, the use of empirical spectrum occupancy data is essential. The development of spectrum occupancy models based on empirical measurements poses significant challenges and opens interesting research problems. This section provides a general overview of this research topic. A more detailed and in-depth discussion can be found in [38]. 
A first and necessary step is obtaining spectrum occupancy data from real systems that can be used in the development of models. A significant number of spectrum occupancy measurements has been performed around the world (see [39] and references therein). However, these measurement studies are based in quite dissimilar measurement platforms and methodologies. The development of a methodology (including the design and implementation of an appropriate measurement platform [40], the realisation of field measurements and the post-processing of the measurement data) that is appropriate for measurements in the context of spectrum models and the harmonisation of methodologies across independent studies (to make results comparable) is a challenging open problem [39].

The development of spectrum occupancy models needs to take into account aspects such as the set of parameters characterised by the model, how such parameters are modelled, the information provided by the model, etc. (see [38] for a more detailed discussion). The following list describes some relevant parameters in the time, frequency and space domains along with some references with the corresponding models. A general overview can be found in $[38,41]$ and a more detailed description and discussion is provided in $[42,43]$.

Time-dimension parameters:

- Channel load (duty cycle): The channel load is a relevant parameter because it determines the amount of spectrum opportunities that a DSA/CR system can expect to find in a channel. The channel load can be characterised in terms of the duty cycle, which represents the fraction of time that the channel is busy. While the average value of this parameter is relevant, its temporal variation pattern also represents an interesting aspect to analyse [44].

- Period durations (minimum, average, variance, distribution, etc.): A more complete and realistic modelling requires not only the duty cycle but also the durations of busy and idle periods to be taken into account. A common assumption in existing literature is that period durations are exponentially distributed. Empirical studies demonstrate that such assumption is inaccurate and other distributions are more appropriate [45].

- Correlation properties of period durations: Two different correlation metrics can be distinguished, namely the correlation between the durations of periods of different type (i.e., busy and idle periods) and the correlation between the sequence of periods of the same type (either busy or idle) and a shifted version of itself (i.e., the autocorrelation), which has experimentally been observed to exhibit two different behaviours (periodic/oscillating and non-periodic/decreasing) [46].

Frequency-dimension parameters:

- $\quad$ Statistical distribution of duty cycle: The duty cycle values for individual channels within the same spectrum band have been shown to follow a beta distribution. The Kumaraswamy distribution, which closely resembles the beta distribution, can also be used as an appropriate model [47].

- Clustering of duty cycle: Within a spectrum band, channels with similar load/duty cycle values are not found alone but in groups (clusters) of a certain size (i.e., the duty cycle is clustered over frequency) [47].
Space-dimension parameters:

- Perceived spectrum occupancy level: DSA/CR users at different locations observe different spectrum occupancy patterns depending on the surrounding environment and radio propagation conditions [48]. Spatial models are aimed at describing/predicting how the spectrum occupancy perceived by DSA/CR users depends on the user location $[49,50]$.

Spectrum occupancy modelling has received an increasing interest recently. However, existing spectrum models do not cover the whole range of possible scenarios of interest and more comprehensive modelling solutions are required. Spectrum occupancy modelling in the context of DSA/CR still requires more work to be done.

\section{DISCUSSION}

Owing to the opportunistic nature of DSA/CR, the behaviour and performance of a DSA/CR system is highly dependent on the surrounding radio environment. For this reason, spectrum awareness is a function of paramount importance in DSA/CR systems. In its broadest sense, the purpose of the spectrum awareness function is to provide a DSA/CR system with relevant information and knowledge on the surrounding radio environment (this may include a broad range of information types). In the context of DSA/CR, this is commonly understood as the identification of portions of the radio spectrum that are available for opportunistic use (spectrum holes or white spaces [17]) at any time, which can be performed by means of spectrum sensing. However, the binary idle/busy information provided by spectrum sensing can be further processed in order to gain a more detailed and sophisticated knowledge of the environment, including statistical information on the spectral activity of a primary system in a certain spectrum band and the inference of the spectrum occupancy patterns based on spectrum occupancy models. This information can be extremely valuable for DSA/CR systems since it can be exploited in spectrum and radio resource decision-making processes, leading to an increased DSA/CR system performance, a better protection of primary licensed systems from potentially harmful interference, and a more efficient use of the precious spectral resources.

This paper has provided an overview not only on relevant spectrum awareness methods (in general) and spectrum sensing algorithms (in particular), but also on how the basic idle/busy information provided by spectrum sensing can be further processed in order to elaborate more sophisticated information. More concretely, this paper has reviewed the following relevant problems in the context of spectrum awareness in DSA/CR systems: i) spectrum sensing algorithms, methods and techniques in the context of spectrum occupancy estimation; ii) reliable and accurate estimation of spectrum occupancy statistics based on empirical observations (i.e., spectrum sensing); and iii) statistical models for characterising spectrum occupancy patterns. It is worth noting that each of these problems rely on the previous one (i.e., spectrum sensing provides basic idle/busy information, which is further elaborated to estimate the channel activity statistics, and such channel activity statistics can be used to parametrise more sophisticated spectrum occupancy models). Existing solutions for each of these problems have been reviewed and open research problems have been mentioned as well. 


\section{REFERENCES}

[1] C. Jackson, "Dynamic sharing of radio spectrum: A brief history," in Proc. First IEEE Int'l. Symp. Dyn. Spect. Access Networks (DySPAN 2005), Nov. 2005, pp. 445-466.

[2] Q. Zhao and B. M. Sadler, "A survey of dynamic spectrum access," IEEE Signal Process. Mag., vol. 24, no. 3, pp. 79-89, May 2007.

[3] M. M. Buddhikot, "Understanding dynamic spectrum access: Taxonomy, models and challenges," in Proc. 2nd IEEE Int'l. Symp. Dyn. Spect. Access Networks (DySPAN 2007), Apr. 2007, pp. 649-663.

[4] Y.-C. Liang, K.-C. Chen, G. Y. Li, and P. Mähönen, "Cognitive radio networking and communications: An overview," IEEE Trans. Vehic. Tech., vol. 60, no. 7, pp. 3386-3407, Sep. 2011.

[5] M. López-Benítez, "Cognitive radio," in Heterogeneous cellular networks: Theory, simulation and deployment. Cambridge University Press, 2013, ch. 13, pp. 383-425.

[6] S. Yarkan and H. Arslan, "Binary time series approach to spectrum prediction for cognitive radio," in Proc. IEEE 66th Vehic. Tech. Conf. (VTC 2007 Fall), Sep. 2007, pp. 1563-1567.

[7] V. K. Tumuluru, P. Wang, and D. Niyato, "A neural network based spectrum prediction scheme for cognitive radio," in Proc. 2010 IEEE Int'l. Conf. Comms. (ICC 2010), May 2010, pp. 1-5.

[8] X. Liu, B. Krishnamachari, and H. Liu, "Channel selection in multichannel opportunistic spectrum access networks with perfect sensing," in Proc. 2010 IEEE Int'l. Symp. Dyn. Spect. Access Networks (DySPAN 2010), Apr. 2010, pp. 1-8.

[9] J. Vartiainen, M. Höyhtyä, J. Lehtomäki, and T. Bräysy, "Priority channel selection based on detection history database," in Proc. Fifth Int'l. Conf. Cognitive Radio Oriented Wireless Networks \& Comms. (CROWNCOM 2010), Jun. 2010, pp. 1-5.

[10] M. Höyhtyä, S. Pollin, and A. Mämmelä, "Classification-based predictive channel selection for cognitive radios," in Proc. 2010 IEEE Int'l. Conf. Comms. (ICC 2010), May 2010, pp. 1-6.

[11] F. Bouali, O. Sallent, J. Pérez-Romero, and R. Agustí, "Strengthening radio environment maps with primary-user statistical patterns for enhancing cognitive radio operation," in Proc. Sixth Int'l. ICST Conf. Cognitive Radio Oriented Wireless Networks and Comms. (CROWNCOM 2011), Jun. 2011, pp. 256-260.

[12] E. Jung and X. Liu, "Opportunistic spectrum access in multiple-primaryuser environments under the packet collision constraint," IEEE/ACM Trans. Networking, vol. 20, no. 2, pp. 501-514, Apr. 2012.

[13] S. Geirhofer, L. Tong, and B. M. Sadler, "Cognitive medium access: constraining interference based on experimental models," IEEE J. Sel. Areas Comms., vol. 26, no. 1, pp. 95-105, Jan. 2008.

[14] J. Jia, Q. Zhang, and X. Shen, "HC-MAC: A hardware-constrained cognitive MAC for efficient spectrum management," IEEE J. Sel. Areas Comms., vol. 26, no. 1, pp. 106-117, Jan. 2008.

[15] H. Su and X. Zhang, "Cross-layer-based opportunistic MAC protocols for QoS provisioning over cognitive radio wireless networks," IEEE $J$. Sel. Areas Comms., vol. 26, no. 1, pp. 118-129, Jan. 2008

[16] A. Anandkumar, N. Michael, A. K. Tang, and A. Swami, "Distributed algorithms for learning and cognitive medium access with logarithmic regret," IEEE J. Sel. Areas Comms., vol. 29, no. 4, pp. 731-745, 2011.

[17] R. Tandra, A. Sahai, and S. M. Mishra, "What is a spectrum hole and what does it take to recognize one?" Proc. IEEE, vol. 97, no. 5, pp. 824-848, May 2009.

[18] M. M. Buddhikot, P. Kolodzy, S. M. adn K. Ryan, and J. Evans, "DIMSUMnet: new directions in wireless networking using coordinated dynamic spectrum," in Proc. 6th IEEE Int'l. Symp. World of Wireless Mob. \& Multimedia Netws. (WoWMoM 2005), June 2005, pp. 78-85.

[19] D. Raychaudhuri and X. Jing, "A spectrum etiquette protocol for efficient coordination of radio devices in unlicensed bands," in Proc. 14th IEEE Int'l. Symp. Pers., Indoor and Mobile Radio Comms. (PIMRC 2003), vol. 1, September 2003, pp. 172-176.

[20] P. Houze, S. B. Jemaa, and P. Cordier, "Common pilot channel for network selection," in Proc. IEEE 63rd Vehic. Tech. Conf. (VTC 2006Spring), vol. 1, May 2006, pp. 67-71.

[21] O. Sallent, J. Pérez-Romero, R. Agustí, and P. Cordier, "Cognitive pilot channel enabling spectrum awareness," in Proc. IEEE Int'l. Conf. Comms. Workshops (ICC Workshops 2009), June 2009, pp. 1-6.

[22] M. Filo, A. Hossain, A. R. Biswas, and R. Piesiewicz, "Cognitive pilot channel: enabler for radio systems coexistence," in Proc. Second Int'l. Workshop Cognitive Radio and Advanced Spectrum Management (CogART 2009), June 2009, pp. 17-23.

[23] A. Ghasemi and E. S. Sousa, "Spectrum sensing in cognitive radio networks: requirements, challenges and design trade-offs," IEEE Communications Magazine, vol. 46, no. 4, pp. 32-39, April 2008.

[24] J. Ma, G. Y. Li, and B. H. Juang, "Signal processing in cognitive radio," Proc. IEEE, vol. 97, no. 5, pp. 805-823, May 2009.
[25] Y. Zeng, Y.-C. Liang, A. T. Hoang, and R. Zhang, "A review on spectrum sensing techniques for cognitive radio: Challenges and solutions," EURASIP J. Advances Signal Proces., vol. 2010, pp. 1-15, Jan. 2010.

[26] T. Yücek and H. Arslan, "A survey of spectrum sensing algorithms for cognitive radio applications," IEEE Communications Surveys and Tutorials, vol. 11, no. 1, pp. 116-130, First Quarter 2009.

[27] D. D. Ariananda, M. K. Lakshmanan, and H. Nikookar, "A survey on spectrum sensing techniques for cognitive radio," in Proc. 2nd Int'l. Workshop Cognitive Radio and Advanced Spectrum Management (CogART 2009), May 2009, pp. 74-79.

[28] D. Noguet et al., "Sensing techniques for cognitive radio - state of the art and trends," October 2009, IEEE SCC 41 P1900.6 White paper.

[29] H. Urkowitz, "Energy detection of unknown deterministic signals," Proc. IEEE, vol. 55, no. 4, pp. 523-531, April 1967.

[30] R. Price and N. Abramson, "Detection theory," IEEE Transactions on Information Theory, vol. 7, no. 3, pp. 135-139, July 1961.

[31] J. G. Proakis, Digital communications, 5th ed. McGraw-Hill, 2008.

[32] A. Sahai, N. Hoven, and R. Tandra, "Some fundamental limits on cognitive radio," in Proc. 42nd Allerton Conf. Comms., Control, and Computing (Allerton Conference 2004), October 2004, p. 10.

[33] W. A. Gardner, "Signal interception: a unifying theoretical framework for feature detection," IEEE Transactions on Communications, vol. 36, no. 8, pp. 897-906, August 1988.

[34] W. A. Gardner and C. M. Spooner, "Signal interception: performance advantages of cyclic-feature detectors," IEEE Transactions on Communications, vol. 40, no. 1, pp. 149-159, January 1992.

[35] Y. Zeng and Y.-C. Liang, "Spectrum-sensing algorithms for cognitive radio based on statistical covariances," IEEE Transactions on Vehicular Technology, vol. 58, no. 4, pp. 1804-1815, May 2009.

[36] M. López-Benítez and F. Casadevall, "Improved energy detection spectrum sensing for cognitive radio," IET Communications, Special Issue on Cognitive Communications, vol. 6, no. 8, pp. 785-796, May 2012.

[37] M. López-Benítez, "Can primary activity statistics in cognitive radio be estimated under imperfect spectrum sensing?" in Proc. 24th Annual IEEE Int'l. Symp. Pers., Indoor and Mobile Radio Comms. (PIMRC 2013), Sep. 2013, pp. 750-755.

[38] M. López-Benítez and F. Casadevall, "Spectrum usage in cognitive radio networks: From field measurements to empirical models," IEICE Trans. Comms., Special Section on Technologies for Effective Utilization of Spectrum White Space, vol. E97-B, no. 2, pp. 1-9, Feb 2014.

[39] _ "Methodological aspects of spectrum occupancy evaluation in the context of cognitive radio," European Trans. Telecomms., vol. 21, no. 8, pp. 680-693, Dec. 2010

[40] - "A radio spectrum measurement platform for spectrum surveying in cognitive radio," in Proc. 7th Int'l. ICST Conf. Testbeds \& Res. Infrastr. for the Dev. of Netws. and Communities (TridentCom 2011), Apr. 2011, pp. 59-70.

[41] _ "An overview of spectrum occupancy models for cognitive radio networks," in Proc. IFIP 6th Int'l. Conf. Netw. (NETWORKING 2011), May 2011, pp. 32-41.

[42] M. López-Benítez, "Spectrum usage models for the analysis, design and simulation of cognitive radio networks," Ph.D. dissertation, Dept. Signal Theory and Comms., Universitat Politècnica de Catalunya, 2011.

[43] M. López-Benítez and F. Casadevall, "Spectrum usage models for the analysis, design and simulation of cognitive radio networks," in Cognitive radio and its application for next generation cellular and wireless networks. Springer, 2012, ch. 2, pp. 27-73.

[44] — - "Empirical time-dimension model of spectrum use based on a discrete-time Markov chain with deterministic and stochastic duty cycle models," IEEE Trans. Vehic. Tech., vol. 60, no. 6, pp. 2519-2533, 2011.

[45] - "Time-dimension models of spectrum usage for the analysis, design and simulation of cognitive radio networks," IEEE Trans. Vehic. Tech., vol. 62, no. 5, pp. 2091-2104, Jun. 2013.

[46] — - "Modeling and simulation of time-correlation properties of spectrum use in cognitive radio," in Proc. 6th Int'l. ICST Conf. on Cognitive Radio Oriented Wireless Netws. (CrownCom 2011), Jun. 2011, pp. 1-5.

[47] M. López-Benítez, F. Casadevall, D. López-Pérez, and A. V. Vasilakos, "Modeling and simulation of joint time-frequency properties of spectrum usage in cognitive radio," in Proc. 4th Int'l. Conf. Cognitive Radio and Adv. Spect. Management (CogART 2011), Oct. 2011, pp. 1-5.

[48] M. López-Benítez and F. Casadevall, "On the spectrum occupancy perception of cognitive radio terminals in realistic scenarios," in Proc. 2nd Int'l. Workshop Cog. Inf. Proc. (CIP 2010), Jun. 2010, pp. 1-6.

[49] - "Spatial duty cycle model for cognitive radio," in Proc. 21st IEEE Int'l. Symp. Pers., Indoor and Mobile Radio Comms. (PIMRC 2010), Sep. 2010, pp. 1631-1636.

[50] — "Statistical prediction of spectrum occupancy perception in dynamic spectrum access networks," in Proc. IEEE Int'l. Conf. Comms. (ICC 2011), Jun. 2011, pp. 1-6. 